\title{
Is it time to digitally enable dentistry with the rest of healthcare?
}

On 26 July 2017, the Dental Leadership Clinical Fellows at Health Education England held the first ever Digitally Enabling Dentistry Conference to discuss the art of the possible of how to digitally integrate dentistry with the rest of healthcare. The conference highlighted how other areas of healthcare are collaborating to support the digital agenda set by the NHS Five Year Forward View and the Wachter Review.

The event highlighted how dentists should:

- Have shared access to Electronic Patient Records (EPR) with GPs

- Be able to view patient Summary Care Records (SCR)

- Electronically prescribe to pharmacies via the Electronic Prescribing Service (EPS)

- Have an intelligent dental Electronic Referral System (ERS).

Pharmacy demonstrated how they have implemented SCR into community settings, improving the quality and safety of patient care, as well as integrating with GP practices to provide electronic prescribing (EPS). GP practices have data sharing agreements allowing seamless and secure sharing of patient records with neighbouring GP practices and services.

Camden CCG and South London \& Maudsley NHS Trust (SLAM) proved the power of Interoperability of shared records. SLAM having implemented a cloud based EPR system allow their staff access to patient records from mobile devices regardless of the location and environment their patients are in. Neighbouring boroughs such as Lambeth and Southwark can also view these as part of the Local Care Record for South London. SLAM are in discussion with local dental hospitals to ensure that dentistry is included within this platform.

The challenges and barriers to digitisation were highlighted including addressing digital
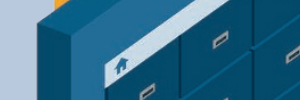

literacy and collaboration as part of professional transformation. The event offered CPD in information governance, cybersecurity and the new general data protection regulation (GDPR) as part of the IG toolkit. The day concluded with innovative dentists and doctors demonstrating how apps and innovation can improve the patient and clinician experience.

The next step is to support our digital dental leaders in driving forward an integrated dental health service and tackle silo working which is further dividing dentistry from the rest of healthcare. The profession has mastered digitisation in their clinical ability, but the digital age ALSO means shared respon-

sibility between healthcare providers including continuity of care, management and logistics that facilitate rapid decision making when it needed. A digitally integrated health service is on the horizon and now is the time to digitally enable dentistry for the future.
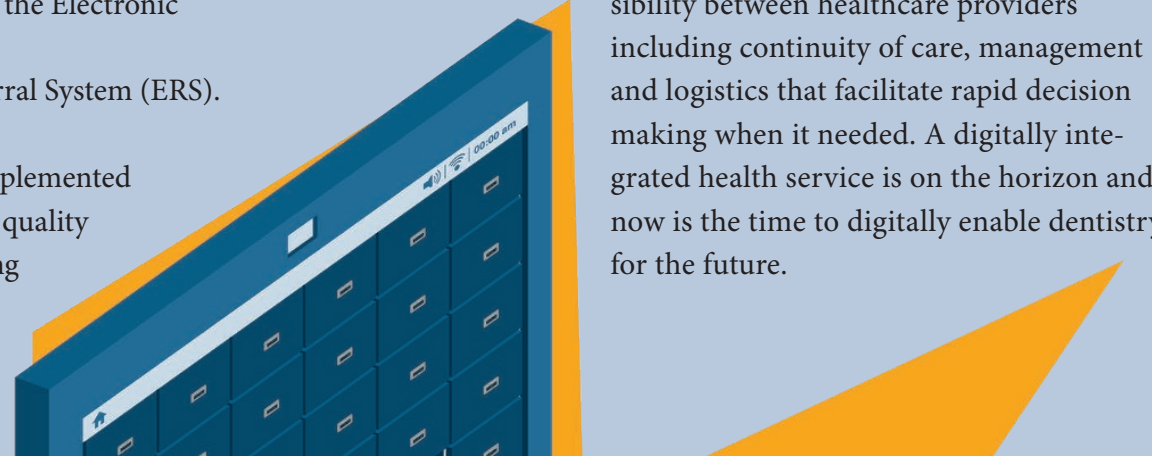